\title{
"GrimAge," an epigenetic predictor of mortality, is accelerated in major depressive disorder
}

\author{
Ekaterina Protsenko $\mathbb{1}^{1}$, Ruoting Yang $\mathbb{0}^{2}$, Brent Nier $^{3}$, Victor Reus ${ }^{3}$, Rasha Hammamieh², Ryan Rampersaud ${ }^{3}$, \\ Gwyneth W. Y. Wu ${ }^{3}$, Christina M. Hough ${ }^{3,4}$, Elissa Epel ${ }^{3}$, Aric A. Prather ${ }^{3}$, Marti Jett $\mathbb{1}^{5}$, Aarti Gautam², \\ Synthia H. Mellon ${ }^{6}$ and Owen M. Wolkowitz $\mathbb{1 0}^{3}$
}

\begin{abstract}
Major depressive disorder (MDD) is associated with premature mortality and is an independent risk factor for a broad range of diseases, especially those associated with aging, such as cardiovascular disease, diabetes, and Alzheimer's disease. However, the pathophysiology underlying increased rates of somatic disease in MDD remains unknown. It has been proposed that MDD represents a state of accelerated cellular aging, and several measures of cellular aging have been developed in recent years. Among such metrics, estimators of biological age based on predictable age-related patterns of DNA methylation (DNAm), so-called 'epigenetic clocks', have shown particular promise for their ability to capture accelerated aging in psychiatric disease. The recently developed DNAm metric known as 'GrimAge' is unique in that it was trained on time-to-death data and has outperformed its predecessors in predicting both morbidity and mortality. Yet, GrimAge has not been investigated in MDD. Here we measured GrimAge in 49 somatically healthy unmedicated individuals with MDD and 60 age-matched healthy controls. We found that individuals with MDD exhibited significantly greater GrimAge relative to their chronological age ('AgeAccelGrim') compared to healthy controls $(p=0.001)$, with a median of 2 years of excess cellular aging. This difference remained significant after controlling for sex, current smoking status, and body-mass index $(p=0.015)$. These findings are consistent with prior suggestions of accelerated cellular aging in MDD, but are the first to demonstrate this with an epigenetic metric predictive of premature mortality.
\end{abstract}

\section{Introduction}

Major depressive disorder (MDD), the leading cause of disability worldwide ${ }^{1}$, is associated with early mortality ${ }^{2}$ and is an independent risk factor for a variety of diseases associated with the aging process ${ }^{3}$, such as cardiovascular disease $^{4-7}$, dementia ${ }^{8}$, osteoporosis ${ }^{9}$, and diabetes ${ }^{10}$, among others. Even after accounting for lifestyle factors, MDD remains an independent risk factor, raising the possibility of an underlying mechanism of accelerated biological or cellular aging ${ }^{3,11}$.

Correspondence: Ekaterina Protsenko (Katerina.protsenko@ucsf.edu) ${ }^{1}$ University of California San Francisco (UCSF) School of Medicine, San Francisco, CA, USA

${ }^{2}$ Medical Readiness Systems Biology, Center for Military Psychiatry and Neuroscience, Walter Reed Army Institute of Research, Silver Spring, MD, USA Full list of author information is available at the end of the article
Recently, epigenetic age has emerged as an especially promising measure of cellular aging that may show stronger associations with mortality than earlier metrics of biological age, such as leukocyte telomere length ${ }^{12}$. It is based on the finding that chronological age has predictable effects on DNA methylation patterns at subsets of the 28 million $5^{\prime}$-C-phosphate-G-3' (CpG) sites scattered throughout the genome ${ }^{13}$. 'Epigenetic clocks' consist of small sets of these CpGs whose methylation patterns yield an estimate of biological or cellular age ('DNAm Age'), as opposed to strictly chronological age. The specific selections of CpGs vary between the available 'clocks,' and are a function of what data the machine learning-derived metric was trained on. When an individual's 'DNAm Age' exceeds their chronological age, they are said to experience 'Epigenetic Age Acceleration.' However, most measures of

\section{(c) The Author(s) 2021}

(c) Open Access This article is licensed under a Creative Commons Attribution 4.0 International License, which permits use, sharing, adaptation, distribution and reproduction cc) in any medium or format, as long as you give appropriate credit to the original author(s) and the source, provide a link to the Creative Commons license, and indicate if changes were made. The images or other third party material in this article are included in the article's Creative Commons license, unless indicated otherwise in a credit line to the material. If material is not included in the article's Creative Commons license and your intended use is not permitted by statutory regulation or exceeds the permitted use, you will need to obtain permission directly from the copyright holder. To view a copy of this license, visit http://creativecommons.org/licenses/by/4.0/. 
'DNAm Age' and 'DNAm Age Acceleration' are only modest predictors of mortality, likely because their derivation was based on chronological age, which by its definition excluded those CpG sites that would signal a departure from normal aging ${ }^{14}$.

In an effort to improve upon mortality prediction, the 'GrimAge' clock used a two-stage approach to derive its algorithm $^{15}$. In the first stage, CpG sites were identified whose methylation states closely correlated with either serum protein levels that predicted mortality, or with selfreported smoking history. In the second stage, these 'DNAm surrogates' for serum proteins and smoking history were further trained on large-scale time-to-death data. Not surprisingly, GrimAge outperformed its predecessors in its ability to predict mortality in five independent cohorts $^{15}$. It was also able to predict time to onset of coronary heart disease, and has shown associations with congestive health failure, hypertension, type- 2 diabetes, physical functioning, comorbidity, and early menopause $^{15}$. In all, because GrimAge's component CpG sites are surrogates for health-related and disease-related proteins, as well as smoking history, it demonstrates superior associations with all-cause mortality and agerelated health status ${ }^{16}$, and is therefore likely to be more sensitive for accelerated cellular aging in psychiatric conditions than its predecessors.

Despite the consensus that depression is associated with premature morbidity and mortality, there have been very few published investigations into potential changes in epigenetic aging in $\mathrm{MDD}^{17}$, and none using the GrimAge metric. In light of the relative dearth of evidence on epigenetic aging in MDD, we investigated GrimAge Acceleration ('AgeAccelGrim') in a cohort of somatically healthy, unmedicated individuals with moderate-to-severe MDD, compared to a group of medically and psychiatrically healthy controls. We hypothesized, based on evidence of early mortality among depressed individuals, that GrimAge Acceleration would be greater in MDD than in similarly aged healthy controls.

\section{Methods}

\section{Ethics statement}

The University of California, San Francisco (UCSF) Institutional Review Board (IRB) approved the study protocol. All study participants gave written informed consent to participate in this study and were compensated for participating.

\section{Recruitment procedures and study participants}

$\operatorname{MDD}(n=50)$ outpatients and healthy controls $(n=63)$ were recruited by flyers, Craigslist postings, newspaper ads and, in the case of MDD subjects, clinical referrals. All diagnoses, including MDD, were made according to DSM-IV guidelines ${ }^{18}$, which were in use at the start of this study. Diagnoses were established using the Structured Clinical Interview for DSM-IV $\mathrm{TR}^{19}$ and verified in a separate unstructured diagnostic evaluation by a Boardcertified psychiatrist. Depression symptom severity was assessed in MDD subjects using the Hamilton Depression Rating Scale $(\mathrm{HDRS})^{20}$, while depression severity across MDD and control participants was assessed with the selfrated Inventory of Depressive Symptoms (IDS-SR) ${ }^{21}$. All MDD subjects had a minimum 17-item HDRS score of 17 . MDD subjects were excluded if they met DSM-IV criteria for any of the following: (i) bipolar disorder, (ii) alcohol or substance abuse within the preceding 6 months, (iii) PTSD or an eating disorder within 1 month of entering the study, and (iv) for any history of psychosis outside of a major depressive episode, or the presence of any psychotic symptoms during the current major depressive episode. Potential healthy controls were excluded for any history of DSM-IV Axis-I diagnoses. All study participants were free of chronic illnesses or acute illnesses or infections, inflammatory disorders, neurological disorders, or any other medical conditions considered to be potentially confounding, as assessed by history, physical examinations, and routine blood screening. All subjects were free of psychotropic medications (including antidepressants), hormone supplements, steroid-containing birth control or other potentially interfering medications, including vitamin supplements above the U.S. recommended daily allowances (e.g. $>90 \mathrm{mg} /$ day for Vitamin C) and had not had any vaccinations for at least 6 weeks prior to enrollment. For MDD subjects, short-acting sedative-hypnotics were allowed as needed up to a maximum of three times per week, but none within 1 week prior to blood draws in the study. All subjects had to pass a urine toxicology screen for drugs of abuse and a urine test for pregnancy in women of child-bearing age on the day of blood draw.

\section{DNA preparation and analysis of methylation}

Blood samples were drawn in the morning following an overnight fast. Whole blood was collected in acid citrate dextrose (ACD) tubes for preparation of DNA. Aliquotted samples were stored frozen at $-80^{\circ} \mathrm{C}$ until use. DNA was extracted from whole blood using QIAmp DNA purification kits (Qiagen, Redwood City, CA), followed by quality check using a Tapestation (Agilent). Identification and analysis of methylated CpGs used protocols used by our group previously ${ }^{22}$. Genomic DNA (500 ng) was treated with sodium bisulfite using the Zymo EZ96 DNA Methylation Kit (Zymo Research, Orange, CA, USA), and genome-wide DNA methylation patterns were profiled using the Infinium HumanMethylation450 BeadChip array (Illumina, Inc., San Diego, CA, USA). BeadChips were washed, single-base extension labeled and stained with multiple layers of fluorescence followed by scanning using the Illumina iScan system (Illumina Inc, CA). The samples 
were randomized on plates. No visual batch effects were found between the plates. IDAT files containing the raw intensity signals were generated using Illumina's iControl software. Noob background correction ${ }^{23}$ was used to preprocess the data prior to submitting it to the DNAm age website https://dnamage.genetics.ucla.edu for analysis. A principal component analysis (PCA) was conducted on Noob-corrected data to identify outliers with DNA methylation assay anomalies. Three healthy controls and one MDD were excluded for the quality control. All the samples were processed and assayed together, and PCA analysis confirmed no significant batch effect existed on experimental blood collection batches. All samples passed quality control, and mean absolute difference between samples and the "gold standard" (which is the mean of a large whole-blood DNAm cohort) were all lower than 0.08 .

\section{Epigenetic clocks}

The GrimAge clock has been previously described ${ }^{15}$. 'GrimAge Acceleration' is defined as the residual from regressing epigenetic age on chronological age, and is denoted by the prefix "AgeAccel" (e.g., "AgeAccelGrim"). The GrimAge clock is constructed as the composite of 8 DNA methylation-based markers for plasma proteins and self-reported smoking packyears ${ }^{15}$. The plasma protein surrogates include: cystatin $\mathrm{C}$, leptin, tissue inhibitor metalloproteinases 1 (TIMP1), adrenomedullin (ADM), beta-2-microglobulin (B2M), growth differentiation factor 15 (GDF15), and plasminogen activation inhibitor 1 (PAI1). The rationale for selection of these proteins, and their functions and disease-associations are described in $\mathrm{Lu}$ et al. (2019) (see ref. ${ }^{15}$ ). The DNAm surrogates for these proteins and smoking history are denoted by the prefix "DNAm" (e.g., "DNAmPACKYRS" for the surrogate of smoking history), and the residuals from regressing on chronological age are specified as "age-adjusted."

\section{Statistical methods}

The sample size included was based on all subjects with available data from the parent study ("Cell Aging in Major Depression"; R01-MH083784). The resulting sample was sufficient to detect effect sizes for independent sample $t$ tests of 0.54 or greater, with a power of 0.80 and a twotailed alpha $=0.05$.

$P$ values for all analyses reflect two-tailed significance with an alpha $=0.05$. Correlations between epigenetic age and chronological age were based on Spearman correlations. All measures of epigenetic age were subsequently regressed on chronological age, and the residuals were used for all ANCOVA analyses. The age-adjusted residual of GrimAge is referred to as "AgeAccelGrim" to maintain consistency with prior literature. All variables were checked for normality by a Shapiro-Wilk test, and Blomtransformation $^{24}$ was used to achieve normality for
AgeAccelGrim. Several of the age-adjusted DNAm surrogate marker components of GrimAge were also Blom-transformed: DNAmCystatinC, DNAmLeptin, DNAmPACKYRS, and DNAmTIMP1. For all sub-cohort analyses, Blom transformation was repeated within the sub-cohort prior to analysis. The remaining age-adjusted DNAm components were normally distributed.

All group differences in age-adjusted epigenetic age metrics were initially tested using independent samples $T$-tests ('Model 1'). Since one of the DNAm surrogate markers included in the GrimAge algorithm was derived nominally as a surrogate for lifetime pack-years of smoking (DNAmPACKYRS $)^{15}$, we covaried for current tobacco use in a separate ANCOVA ('Model 2'). We additionally covaried for sex and BMI in 'Model 3,' as both have been repeatedly shown to modify measures of epigenetic aging ${ }^{15,25-28}$. The same models were applied to the individual GrimAge surrogate marker components. DNAmPACKYRS was analyzed in both its raw and age-adjusted forms.

To control for any lingering effects of smoking not accounted for by covariance, we completed sensitivity analyses within a sub-cohort of 'current non-smokers' (37 MDD, 55 HC; 'Model 4 and 5'). While 'current' smoking status was used in the primary analyses to maximize sample size, additional data on lifetime smoking exposure were available in a subset of participants (41 MDD, 51 HC). Models 2 and 3 for AgeAccelGrim and DNAmPACKYRS were repeated in this subset to ensure the effect did not differ when lifetime smoking was accounted for, defined as 'never,' 'former,' or 'current' smoker ('Model 6 and 7'). Finally, we excluded the DNAmPACKYRS component from AgeAccelGrim, as has been previously described ${ }^{22}$. These analyses are detailed in the Supplementary Materials, except Models 4 and 5 which are detailed below.

\section{Results}

\section{Participant demographics and depressive symptoms}

The MDD and control groups did not differ significantly in chronological age, sex distribution, BMI, race, ethnicity, educational attainment, lifetime history of smoking, or estimated number of lifetime cigarettes among smokers (Table 1). However, the groups did differ in a binary metric of current tobacco use (Yes/No), with greater current smoking reported in the MDD group (chi-square $=6.653$, $p=0.010)$ and, as expected, in scores on the Inventory of Depressive Symptoms (IDS) $(t(54.224)=-20.239, p=$ 0.000 ). Further characterization of participants with MDD is presented in Table 1.

\section{GrimAge acceleration in depressed patients and healthy controls}

GrimAge was significantly correlated with chronological age in the combined cohort (Spearman Rho $=0.968$, 
Table 1 Participant characterization.

\begin{tabular}{|c|c|c|c|}
\hline & $\operatorname{MDD}(N=49)$ & Healthy control $(N=60)$ & \\
\hline Age, years [mean (sd; range)] & $40.2(14.6 ; 20-68)$ & $39.4(13.6 ; 21-65)$ & $t(107)=-0.294, p=0.769$ \\
\hline Body mass index [mean (sd; range)] & $25.8(4.4 ; 19.0-36.3)$ & $24.5(4.8 ; 17.7-41.5)(n=59)$ & $t(106)=-1.463, p=0.146$ \\
\hline Sex Female $[n(\%)]$ & $27(55 \%)$ & $36(60 \%)$ & $\begin{array}{l}\text { chi-square }=0.265 \\
p=0.607\end{array}$ \\
\hline \multicolumn{4}{|l|}{$\operatorname{Race}[n(\%)]$} \\
\hline Caucasian & $29(59 \%)$ & $38(63 \%)$ & \multirow{6}{*}{$\begin{array}{l}\text { chi-square }=4.013 \\
p=0.404\end{array}$} \\
\hline Black or African American & $4(8 \%)$ & $5(8 \%)$ & \\
\hline Asian & $9(18 \%)$ & $12(20 \%)$ & \\
\hline Other & $5(10 \%)$ & $1(2 \%)$ & \\
\hline More than one & $2(4 \%)$ & $4(7 \%)$ & \\
\hline Ethnicity [n(\%)] & $(n=48)$ & $(n=56)$ & \\
\hline Hispanic & $9(19 \%)$ & $6(11 \%)$ & \multirow{3}{*}{$\begin{array}{l}\text { chi-square }=1.352 \\
p=0.245\end{array}$} \\
\hline Non-Hispanic & $39(81 \%)$ & $50(89 \%)$ & \\
\hline Education $[n(\%)]$ & & $(n=59)$ & \\
\hline High School & $3(6 \%)$ & $1(2 \%)$ & \multirow{5}{*}{$\begin{array}{l}\text { chi-square }=8.674 \\
p=0.070\end{array}$} \\
\hline Some College/Tech School & $14(29 \%)$ & $6(10 \%)$ & \\
\hline Associate's Degree & $2(4 \%)$ & $3(5 \%)$ & \\
\hline Bachelor's Degree & $17(35 \%)$ & $32(54 \%)$ & \\
\hline Advanced Degree & $13(26 \%)$ & $17(29 \%)$ & \\
\hline \multicolumn{4}{|l|}{ Smoking history } \\
\hline Current smoker $[n(\%)]$ & $12(24 \%)(n=49)$ & $4(7 \%)(n=59)$ & $\begin{array}{l}\text { chi-square }=\mathbf{6 . 6 5 3} \\
p=\mathbf{0 . 0 1 0}\end{array}$ \\
\hline Ever smoker $[n(\%)]$ & $21(51 \%)(n=41)$ & $21(41 \%)(n=51)$ & $\begin{array}{l}\text { chi-square }=0.924 \\
p=0.336\end{array}$ \\
\hline $\begin{array}{l}\text { Estimated lifetime cigarettes in ever smokers }{ }^{\mathrm{a}} \text { [mean (sd; } \\
\text { range)] }\end{array}$ & $\begin{array}{l}26,859(41,251 ; 20-164,250) \\
(n=21)\end{array}$ & $\begin{array}{l}22,304(46,339 ; 5-164,250) \\
(n=21)\end{array}$ & $U=285.5, p=0.102$ \\
\hline \multicolumn{4}{|l|}{ Characterization of major depressive disorder [mean (sd)] } \\
\hline Depressive Symptom Score (IDS) & $32.5(8.9)$ & $4.2(3.5)$ & $\begin{array}{l}t(54.224)=-\mathbf{2 0 . 2 3 9 ^ { b }} \\
p=\mathbf{0 . 0 0 0}\end{array}$ \\
\hline Hamilton Depression Rating Scale (HDRS) & $20.3(3.5)$ & - & - \\
\hline Duration of Current Depressive Episode (days) ${ }^{c}$ & $1918(3521)$ & - & - \\
\hline Chronicity of Lifetime Depression (months) $^{d}$ & $133(124)(n=48)$ & - & - \\
\hline Number of Depressive Episodes & $4.4(2.9)$ & - & - \\
\hline Lifetime Days of Depression ${ }^{e}$ & $3990(3775)$ & - & - \\
\hline Lifetime Days of Untreated Depression ${ }^{f}$ & 2966 (4661) & - & - \\
\hline
\end{tabular}

p-values reflect 2-tailed significance.

${ }^{a}$ Estimated Lifetime Cigarettes in Ever Smokers-The lifetime number of cigarettes was estimated from self-report by first determining the blocks of time the participant smoked a given number of cigarettes per day, multiplying that number of days by the daily cigarette usage in that block of time, and then summing those products. Periods of non-smoking were not included in the estimate.

${ }^{b} t$-values reflect unequal variance between groups by Levene's Test.

'Duration of Current Depressive Episode-self-reported days since the onset of meeting DSM criteria for an Major Depressive Episode until the date of the evaluation. ${ }^{d}$ Chronicity of Lifetime Depression (months) - self-reported number of months since the onset of the first depressive episode. Includes any periods of remission of symptoms between major depressive episodes for those with more than 1 major depressive episode.

eLifetime Days of Depression-Estimated from detailed psychiatric history of number and duration of major depressive episodes. Does not include periods of remission of symptoms between depressive episodes for those with more than 1 major depressive episode.

fLifetime Days of Untreated Depression-Calculated as the difference between Lifetime Days of Depression and Lifetime Days of Treated Depression, both estimated from detailed psychiatric history. 
$p<0.001$ ), and among participants with MDD and controls separately (MDD: Spearman Rho $=0.974, p<0.001$; HC: Spearman Rho $=0.961, p<0.001$ ) (Fig. 1B), consistent with strong correlations reported previously ${ }^{15}$ and with the inclusion of chronological age as a component of the GrimAge algorithm.

In our primary analysis, participants with MDD showed significantly greater AgeAccelGrim compared to agematched healthy controls $(t(107)=-3.265, p=0.001$; Cohen's $d=0.63$ ). Based on median values prior to transformation for normality, this equates to 2.0 years of accelerated GrimAge (MDD: median $=0.364$ years, interquartile range $(\mathrm{IQR})=2.180$; $\mathrm{HC}$ : median $=-1.637$ years, interquartile range $(\mathrm{IQR})=3.946)($ Fig. $1 \mathrm{~A})$. The group effect remained statistically significant after adjustment for current smoking (Model 2: $F_{\mathrm{MDD}}(1,109)=$ 7.345, $p=0.008)$, and after full adjustment for smoking, sex, and BMI (Model 3: $F_{\mathrm{MDD}}(1,103)=6.095, p=0.015$ ) (Table 2).

Although AgeAccelGrim was significantly higher in MDDs vs. controls, it was not significantly correlated with scores of depression severity on the 17-item Hamilton Depression Rating Scale (HDRS) $(r=-0.088, p=0.550)$ within the MDD group, nor was it associated with reported lifetime days of depression or days of untreated depression (lifetime days: $r=-0.009, p=0.953$; untreated days: $r=0.061, p=0.676$ ), chronicity of lifetime depression $(r=-0.014, p=0.926)$, or duration of the current depressive episode $(r=-0.125, p=0.391)$ (see Table 1 for variable definitions).

\section{Individual GrimAge component markers and the role of DNAmPACKYRS}

Among the benefits of the GrimAge clock is that each of its surrogate DNAm markers can themselves be queried as a means to explore their contribution to accelerated cellular aging ${ }^{15}$. Of the individual surrogates, only ageadjusted DNAmCystatinC and raw and age-adjusted DNAmPACKYRS significantly differed between MDD and healthy control participants (Table 2). The group differences in age-adjusted DNAmCystatinC survived adjustment for smoking, but missed statistical significance after full adjustment for smoking/sex/BMI. In contrast, the group difference in raw and age-adjusted DNAmPACKYRS survived full adjustment (Table 2).

Notably, while the DNAmPACKYRS metric was developed as a surrogate for self-reported smoking history ${ }^{15}$, the $172 \mathrm{CpGs}$ of which it consists are related to a number of other functions ${ }^{29,30}$. To explore whether the DNAmPACKYRS metric captures some underlying biological aspect of aging that is not specific to smoking, we (1) restricted our between-group analysis of AgeAccelGrim and DNAmPACKYRS to current non-smokers in both the MDD and control groups, and (2) covaried for trilevel 'never,' 'former,' and 'current' smoking status in a subcohort of participants with detailed data on smoking exposure. Among current non-smokers, we still found a statistically significant difference between the MDD and healthy control groups in the overall AgeAccelGrim (Model 4: $t(89.68)=-2.383, p=0.019$, Cohen's $d=0.49$ ) and in both raw and age-adjusted DNAmPACKYRS
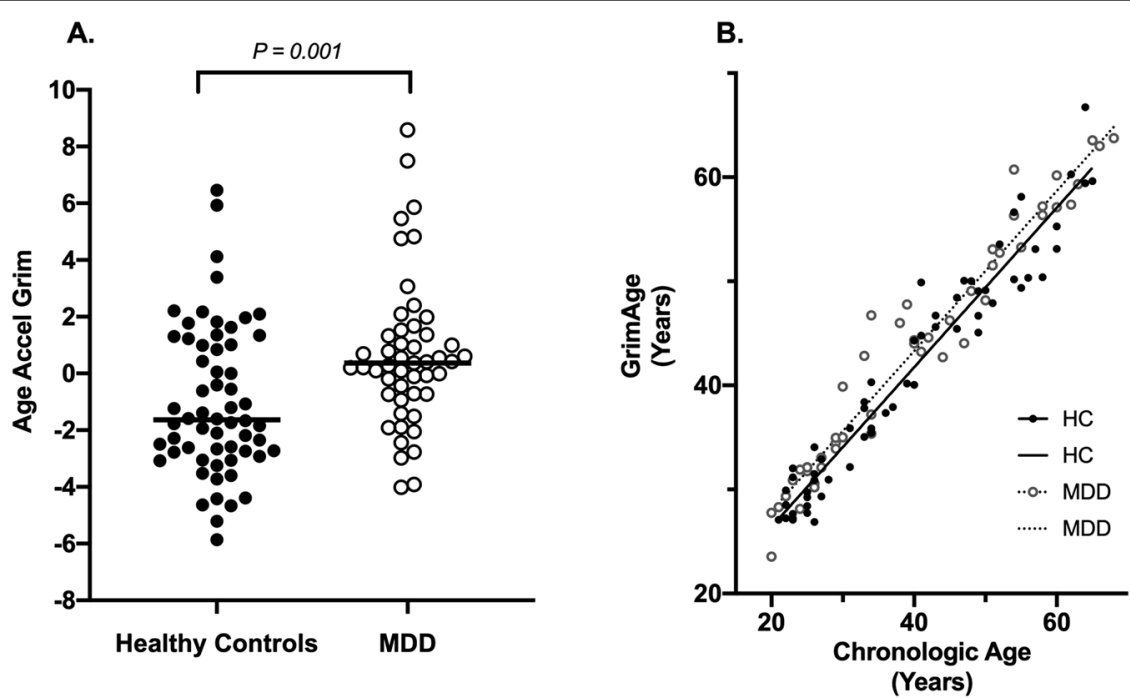

Fig. 1 Cross-section differences in AgeAccelGrim between healthy controls and patients with major depressive disorder (MDD). A Plotted values are raw AgeAccelGrim measures (in years), prior to Blom transformation. $p$-value reflects two-tailed significance between groups, based on Blom-transformed data to achieve normality of distribution. Horizontal line indicates median AgeAccelGrim within each group. $N_{\text {Healthy Control }}=60$, $N_{\mathrm{MDD}}=49$. B Plotted values are participants' chronological age plotted against GrimAge (prior to age-adjustment), demonstrating a strong correlation between chronological age and GrimAge among both participants with MDD and healthy controls (HC) (Combined: Spearman $R h o=0.968, p<0.001$; MDD: Spearman Rho $=0.974, p<0.001$; HC: Spearman Rho $=0.961, p<0.001$ ). 
Table 2 GrimAge Clock and its components in MDD and HC.

\begin{tabular}{|c|c|c|c|c|c|c|c|c|c|}
\hline \multirow[t]{2}{*}{ MDD vs. Control: } & \multirow{2}{*}{$\begin{array}{l}\text { MDD: } N=49 \\
\text { Mean } \pm \text { SD }\end{array}$} & \multirow{2}{*}{$\begin{array}{l}\text { Control: } N=60 \\
\text { Mean } \pm \text { SD }\end{array}$} & \multicolumn{3}{|l|}{ Model $1^{\mathrm{a}}$} & \multicolumn{2}{|l|}{ Model $2^{b}$} & \multicolumn{2}{|l|}{ Model $3^{c}$} \\
\hline & & & $t(\mathrm{df}=107)$ & $p$-value & Cohen's $d$ & $\begin{array}{l}F_{\mathrm{MDD}} \\
(\mathrm{df}, \mathrm{df}=1,105)\end{array}$ & $p$-value & $\begin{array}{l}F_{M D D} \\
(d f, d f=1,103)\end{array}$ & $p$-value \\
\hline \multicolumn{10}{|c|}{ Age-Adjusted GrimAge (aka "AgeAccelGrim") } \\
\hline AgeAccelGrim $^{\mathrm{d}}$ & $0.30 \pm 0.84$ & $-0.29 \pm 1.01$ & -3.265 & 0.001 & 0.63 & 7.345 & 0.008 & 6.095 & 0.015 \\
\hline \multicolumn{10}{|c|}{ Age-Adjusted Epigenetic Components of GrimAge } \\
\hline DNAmPACKYRS ${ }^{d}$ & $0.30 \pm 0.87$ & $-0.30 \pm 1.00$ & -3.306 & 0.001 & 0.64 & 7.042 & 0.009 & 6.668 & 0.011 \\
\hline DNAmCystatin $C^{d}$ & $0.18 \pm 0.84$ & $-0.23 \pm 1.04$ & -2.203 & 0.030 & 0.43 & 4.639 & 0.034 & 3.817 & 0.053 \\
\hline DNAmLeptin ${ }^{d}$ & $0.08 \pm 1.01$ & $-0.04 \pm 0.96$ & -0.599 & 0.551 & 0.12 & 0.235 & 0.629 & 1.421 & 0.236 \\
\hline DNAmTIMP $1^{d}$ & $0.12 \pm 0.92$ & $-0.10 \pm 1.04$ & -1.133 & 0.260 & 0.22 & 1.812 & 0.181 & 0.951 & 0.332 \\
\hline DNAmADM & $-0.11 \pm 18.61$ & $0.27 \pm 15.59$ & 0.114 & 0.909 & 0.02 & 0.048 & 0.828 & 0.008 & 0.927 \\
\hline DNAmB2M & $1005.14 \pm 51,306.85$ & $-12,031.01 \pm 69,546.19$ & $-1.125^{\mathrm{e}}$ & 0.263 & 0.21 & 1.877 & 0.174 & 1.677 & 0.198 \\
\hline DNAmGDF15 & $3.21 \pm 52.28$ & $-6.86 \pm 55.64$ & -0.965 & 0.337 & 0.19 & 0.440 & 0.508 & 0.387 & 0.535 \\
\hline DNAmPAl1 & $-35.72 \pm 1916.48$ & $-142.78 \pm 2646.10$ & $-0.245^{\mathrm{e}}$ & 0.807 & 0.05 & 0.041 & 0.840 & 0.150 & 0.699 \\
\hline \multicolumn{10}{|c|}{ Non-Age-Adjusted DNAmPACKYRS } \\
\hline DNAmPACKYRS ${ }^{d}$ & $0.29 \pm 1.00$ & $-0.23 \pm 0.93$ & -2.816 & 0.006 & 0.54 & 5.717 & 0.019 & 4.988 & 0.028 \\
\hline
\end{tabular}

$p$-values reflect 2-tailed significance.

All models used age-adjusted metrics of epigenetic age. Age-adjusted metrics were calculated as the residual from regressing GrimAge and its components on chronological age. DNAmPACKYRS was analyzed in both its age-adjusted and raw forms.

Age-adjusted GrimAge is denoted as "AgeAccelGrim" to maintain consistency with the literature.

Bold indicates statistically significant results, and their associated Cohen's d values where relevant.

${ }^{a}$ Model 1: Independent samples T-test.

${ }^{b}$ Model 2: ANCOVA covaried for current smoking status (binary $\mathrm{Y} / \mathrm{N}$ ).

${ }^{c}$ Model 3: ANCOVA covaried for current smoking status (binary $\mathrm{Y} / \mathrm{N}$ ), sex, and BMI.

${ }^{\mathrm{d} D e n o t e s}$ epigenetic age variables that were Blom-transformed to achieve normal distributions.

${ }^{e} t$-values reflect unequal variance between groups by Levene's Test.

(Model 4: Raw: $t(90)=-2.401, p=0.018$, Cohen's $d=$ 0.51; Age-Adjusted: $t(89.15)=-2.656, p=0.009$, Cohen's $d=0.55$ ) (Table 3). However, with this smaller sample size, the difference in AgeAccelGrim among non-smokers missed statistical significance after full adjustment for sex and BMI (Model 5: $F_{\mathrm{MDD}}(1,88)=3.417, p=0.068$ ), while the differences in raw and age-adjusted DNAmPACKYRS persisted (Model 5: Raw: $F_{\mathrm{MDD}}(1,88)=4.855, p=0.030$; Age-Adjusted: $\left.\quad F_{\mathrm{MDD}}(1,88)=5.796, \quad p=0.018\right)$. Subcohort analyses on the effect of lifetime smoking status, as opposed to current smoking status, are further detailed in the Supplementary Materials. In brief, differences in both AgeAccelGrim and DNAmPACKYRS between MDD and HC persisted when covarying for trilevel lifetime smoking status (Supplementary Table 1).

Of the 172 CpGs included in the DNAmPACKYRS metric, the cg05575921 site, corresponding to the Aryl Hydrocarbon Receptor Repressor (AHRR) gene, was of particular interest in light of recent evidence suggesting a role in post-traumatic stress disorder ${ }^{31,32}$. To explore if the cg05575921 methylation site might be contributing to differences in DNAmPACKYRS between MDD and HC independent of smoking exposure, we assessed differences in cg05575921 methylation (1) associated with smoking and (2) between MDD and HC. These results are presented in the Supplementary Materials, but in brief we did not find differences in cg05575921 methylation between MDD and HC. However, we found significant hypomethylation associated with 'current' smoking status, consistent with prior reports on the cg05575921 site as a marker for smoking exposure ${ }^{33-35}$.

\section{Discussion}

In this paper, we present evidence of epigenetic aging in MDD using the recently developed GrimAge metric, which is highly correlated with morbidity and mortality ${ }^{15}$, in a unique cohort of somatically healthy participants with severe untreated depression. We found that somatically healthy, unmedicated individuals with MDD exhibit greater GrimAge Acceleration than their healthy normal control counterparts, with a median difference of 2 years of accelerated epigenetic aging. Importantly, the differences in AgeAccelGrim between healthy participants and those with MDD persist after adjustment for smoking, sex, and BMI. This suggests that while these variables contribute to the GrimAge, the association with MDD remains above and beyond what is attributable to them.

To date, there have been very few reports on epigenetic aging in MDD, and none specifically on GrimAge in MDD. The most notable of the reports was from the Netherlands Study of Depression and Anxiety ${ }^{17}$. Using an epigenetic age estimate of their own derivation, based on 80,000 CpGs, the group found significant epigenetic age acceleration among depressed patients compared to healthy controls. The effect sizes in both basic and fully adjusted models were small, with Cohen's $d$ of 0.20 and 0.18 , 
Table 3 AgeAccelGrim and DNAmPACKYRS among current non-smokers.

\begin{tabular}{|c|c|c|c|c|c|c|c|}
\hline \multirow[t]{2}{*}{ MDD vs. Control: non-smokers } & \multirow{2}{*}{$\begin{array}{l}\text { MDD: } N=37 \\
\text { Mean } \pm \text { SD }\end{array}$} & \multirow{2}{*}{$\begin{array}{l}\text { Control: } N=55 \\
\text { Mean } \pm \text { SD }\end{array}$} & \multicolumn{3}{|l|}{ Model $4^{\mathrm{a}}$} & \multicolumn{2}{|l|}{ Model $5^{\mathrm{b}}$} \\
\hline & & & $t(\mathrm{df})$ & $p$-value & Cohen's $d$ & $F_{\mathrm{MDD}}(\mathrm{df}, \mathrm{df}=1,88)$ & $p$-value \\
\hline \multicolumn{8}{|c|}{ Age-Adjusted GrimAge (aka "AgeAccelGrim") } \\
\hline AgeAccelGrim $^{c}$ & $0.27 \pm 0.77$ & $-0.18 \pm 1.08$ & $-2.383^{d}(89.68)$ & 0.019 & 0.49 & 3.417 & 0.068 \\
\hline \multicolumn{8}{|c|}{ Age-Adjusted Epigenetic Components of GrimAge } \\
\hline DNAMPACKYRS ${ }^{c}$ & $0.31 \pm 0.78$ & $-0.21 \pm 1.06$ & $-2.656^{d}(89.15)$ & 0.009 & 0.55 & 5.796 & 0.018 \\
\hline \multicolumn{8}{|l|}{ Non-Age-Adjusted DNAmPACKYRS } \\
\hline DNAmPACKYRS & $0.29 \pm 0.92$ & $-0.19 \pm 0.99$ & $-2.401(90)$ & 0.018 & 0.51 & 4.855 & 0.030 \\
\hline
\end{tabular}

respectively, and showed an average 7.68 months of excess epigenetic aging. In our sample, we found significant differences in GrimAge acceleration between MDD participants and healthy controls, with an effect size of Cohen's $d=0.63$ prior to adjustment and a median excess epigenetic aging of 2 years. The greater effect size seen here, if replicated, could be due to the specific epigenetic aging metric used or to differences in our sample characteristics.

In light of the significant between-group differences in current tobacco use and in the DNAmPACKYRS component of the AgeAccelGrim metric, it is important to consider the role of tobacco use in explaining our findings, independent of MDD. To do so, we employed two analytic strategies. We (1) restricted our cohort to 'current non-smokers', and (2) restricted our cohort to subjects with sufficiently detailed smoking history and adjusted for this history. These analyses were consistent with our hypothesis that both AgeAccelGrim and age-adjusted DNAmPACKYRS are associated with MDD independent of smoking. Our pattern of findings is also consistent with those of Lu et al. in their original publication on GrimAge, where they demonstrated that DNAmPACKYRS predicted lifespan in never smokers ${ }^{15}$, suggesting that this composite measure of methylation at $172 \mathrm{CpG}$ sites reflects changes in underlying biological processes not specific to tobacco use. Of these sites, the cg05575921 site and its associated gene-the aryl hydrocarbon receptor repressor (AHRR)-stand out as potentially relevant to the pathophysiology of psychiatric disease $\mathrm{e}^{31,32}$ and aging $^{36,37}$. We compared methylation at this site between MDD and $\mathrm{HC}$ and found no significant differences. Nonetheless, in light of evidence for a role the AHRR in atherosclerosis $^{36,37}$ and PTSD ${ }^{31,32}$ in human studies, in conjunction with evidence from animal studies implicating dysfunction of the AHRR's target-the aryl hydrocarbon receptor-in aging (notably, brain aging) ${ }^{38}$ and neuroinflammation ${ }^{39}$, the AHRR remains an interesting and worthwhile target for further research in depression and other psychiatric diseases.

The strengths of our study include the clinically significant degree of current MDD symptomatology of our sample, the verification of MDD diagnosis by structured and independent unstructured psychiatric interviews, and the requirement that all participants remained medication-free for a minimum of 6 weeks prior to entering the study. Despite the severity of their depression, our sample was otherwise somatically healthy, as we excluded individuals with significant current or past history of medical conditions that would likely themselves affect epigenetic aging. As such, we aimed to evaluate the effects of MDD per se, rather than those of comorbid medical conditions or antidepressant use. However, by so doing we may have selected for a sample of patients that is not fully representative of "general community" depression, who may have biological resilience and have thus experienced fewer somatic sequelae of depression. The same is true of our healthy controls, who were also selected for the absence of somatic illnesses and medication. It appears from the raw data (Fig. 1) that the AgeAccelGrim difference between healthy controls and MDDs is mainly accounted for by lower-than-expected GrimAge among the $\mathrm{HCs}$, rather than by higher-thanexpected GrimAge among MDDs. We interpret this to be due to our careful exclusion of somatic illnesses in both samples, in contrast to the naturalistic community-based samples from which the GrimAge metric was derived.

Limitations of this study include our modest sample size. Our findings therefore require validation in a larger and more diverse cohort. In addition, although participants were largely medication-free for at least 6 weeks prior to 
enrollment, we cannot rule out lingering effects of prior antidepressant or other medication use. We also had detailed data on lifetime smoking history in only a subset of participants, and therefore could only adjust for current smoking status in our primary analysis. While our subcohort analyses demonstrated the effect of MDD on AgeAccelGrim to be robust against adjustment for detailed lifetime smoking exposure, our samples of 'never' smokers were too small to achieve adequate power for analysis and our results will require replication in cohorts with extensive data on smoking exposure or within a large cohort of 'never' smokers. Finally, while the GrimAge metric was shown to predict mortality in other populations ${ }^{15}$, we cannot assert that it indeed does so among individuals with MDD without long-term longitudinal data, or else retrospective data with banked DNA samples.

Individuals with MDD have, on average, lesser life expectancy and greater serious somatic illness comorbidity than non-psychiatrically ill individuals. The reasons for this remain unclear, but our data raise the possibility that the epigenetic changes associated with GrimAge are relevant. However, many questions remain unanswered. Our data do not inform on the stability vs. reversibility of these changes ${ }^{40,41}$, on the actual association of these epigenetic changes with later illness and mortality in MDD, on whether any causal relationships exist between MDD and GrimAge, or on whether the GrimAge differences we noted are specific to MDD among other psychiatric illnesses. Furthermore, while our goal was to assess the possibility of accelerated GrimAging in MDD rather than identify its causes, the ultimate aim of this line of research is to identify the biological mechanisms linking MDD to diminished lifespan and "healthspan"14,15. Advances in understanding the cellular biology underlying serious mental illnesses such as MDD may hold a key to understanding the associated increased risk of illness and death.

\footnotetext{
Acknowledgements

The authors gratefully acknowledge the assistance of Kevin Delucchi, Ph.D., Phuong Hoang, Stacy Ann Miller, the UCSF CTSI Clinical Research Center staff, the PNE Lab research assistants and volunteers, and the research participants. This study was funded by grants from the National Institute of Mental Health (NIMH) (Grant Number R01-MH083784), the O'Shaughnessy Foundation, the Tinberg family, and grants from the UCSF Academic Senate, and the UCSF Research Evaluation and Allocation Committee (REAC). This project was also supported by National Institutes of Health/National Center for Research Resources (NIH/NCRR) and the National Center for Advancing Translational Sciences, NIH, through UCSF-CTSI Grant Numbers UL1 RR024131 and TL-1 TR001871. None of the granting or funding agencies had a role in the design and conduct of the study; collection, management, analysis and interpretation of the data; and preparation, review, or approval of the manuscript. First-author E.P. and co-authors, O.M.W., S.H.M., and R.Y. had full access to all of the data in the study and take responsibility for the integrity of the data and the accuracy of the data analysis. The contents of this publication are solely the responsibility of the authors and do not necessarily represent the official views of the $\mathrm{NIH}$. Material has been reviewed by the Walter Reed Army Institute of Research. There is no objection to its presentation and/or publication. The opinions or assertions contained herein are the private views of the author, and are not to
}

be construed as official, or as reflecting true views of the Department of the Army or the Department of Defense. The investigators have adhered to the policies for protection of human subjects as prescribed in AR 70-25.

\section{Author details}

'University of California San Francisco (UCSF) School of Medicine, San Francisco, CA, USA. ${ }^{2}$ Medical Readiness Systems Biology, Center for Military Psychiatry and Neuroscience, Walter Reed Army Institute of Research, Silver Spring, MD, USA. ${ }^{3}$ Weill Institute for Neurosciences and Department of Psychiatry and Behavioral Sciences, University of California San Francisco (UCSF) School of Medicine, San Francisco, CA, USA. ${ }^{4}$ Department of Psychology, University of California Los Angeles (UCLA), Los Angeles, CA, USA. ${ }^{5}$ US Army MRDC and Walter Reed Army Institute of Research, Silver Spring, MD, USA. ${ }^{6}$ Department of OB-GYN and Reproductive Sciences, University of California San Francisco (UCSF) School of Medicine, San Francisco, CA, USA

Conflict of interest

The authors declare no competing interests.

\section{Publisher's note}

Springer Nature remains neutral with regard to jurisdictional claims in published maps and institutional affiliations.

Supplementary information The online version contains supplementary material available at https://doi.org/10.1038/s41398-021-01302-0.

Received: 16 September 2020 Revised: 21 February 2021 Accepted: 5 March 2021

Published online: 06 April 2021

\section{References}

1. World Health Organization. Depression and Other Common Mental Disorders: Global Health Estimates http://apps.who.int/iris/bitstream/handle/10665/ 254610/WHO-MSD-MER-2017.2-eng.pdf (2017).

2. Walker, E. R., McGee, R. E. \& Druss, B. G. Mortality in mental disorders and global disease burden implications: a Systematic Review and Meta-analysis. JAMA Psychiatry 72, 334 (2015).

3. Wolkowitz, O. M., Reus, V. I. \& Mellon, S. H. Of sound mind and body: depression, disease, and accelerated aging. Dialogues Clin. Neurosci. 13, 25-39 (2011).

4. Schulz, R. et al. Association between depression and mortality in older adults: the Cardiovascular Health Study. Arch. Intern. Med. 160, 1761 (2000).

5. Gan, Y. et al. Depression and the risk of coronary heart disease: a meta-analysis of prospective cohort studies. BMC Psychiatry 14, 371 (2014).

6. Seldenrijk, A. et al. Depression, anxiety and 6-year risk of cardiovascular disease. J. Psychosom. Res. 78, 123-129 (2015).

7. Fiedorowicz, J. G. Depression and cardiovascular disease: an update on how course of illness may influence risk. Curr. Psychiatry Rep. 16, 492 (2014).

8. Green, R. C. et al. Depression as a risk factor for Alzheimer disease: The MIRAGE Study. Arch. Neurol. 60, 753 (2003).

9. Schweiger, U. et al. Low lumbar bone mineral density in patients with major depression. Am. J. Psychiatry 151, 1691-1693 (1994).

10. Evans, D. L. et al. Mood Disorders in the Medically III: scientific review and recommendations. Biol. Psychiatry 58, 175-189 (2005).

11. Wolkowitz, O. M., Epel, E. S., Reus, V. I. \& Mellon, S. H. Depression gets old fast: do stress and depression accelerate cell aging? Depress. Anxiety 27, 327-338 (2010).

12. Gao, X., Zhang, Y., Mons, U. \& Brenner, H. Leukocyte telomere length and epigenetic-based mortality risk score: associations with all-cause mortality among older adults. Epigenetics 13, 846-857 (2018).

13. Horvath, S. \& Raj, K. DNA methylation-based biomarkers and the epigenetic clock theory of ageing. Nat. Rev. Genet. 19, 371-384 (2018).

14. Levine, M. E. et al. An epigenetic biomarker of aging for lifespan and healthspan. Aging 10, 573-591 (2018).

15. Lu, A. T. et al. DNA methylation GrimAge strongly predicts lifespan and healthspan. Aging 11, 303-327 (2019).

16. Hillary, R. F. et al. Epigenetic measures of ageing predict the prevalence and incidence of leading causes of death and disease burden. Clin. Epigenet. 12, 115 (2020). 
17. Han, L. K. M. et al. Epigenetic aging in major depressive disorder. Am. J. Psychiatry 175, 774-782 (2018).

18. American Psychiatric Association. Diagnostic and Statistical Manual of Mental Disorders: DSM-IV-TR (American Psychiatric Association, 2000).

19. First, M. B. SCID-l: Clinician Version (American Psychiatric Press, 1997).

20. Hamilton, M. A rating scale for depression. J. Neurol. Neurosurg. Psychiatry 23, 56-62 (1960).

21. Rush, A. J., Gullion, C. M., Basco, M. R., Jarrett, R. B. \& Trivedi, M. H. The Inventory of Depressive Symptomatology (IDS): psychometric properties. Psychol. Med. 26, 477-486 (1996).

22. PTSD Systems Biology Consortium et al. A DNA methylation clock associated with age-related illnesses and mortality is accelerated in men with combat PTSD. Mol. Psychiatry https://doi.org/10.1038/s41380-020-0755-z (2020).

23. Triche, T. J., Weisenberger, D. J., Van Den Berg, D., Laird, P. W. \& Siegmund, K. D. Low-level processing of Illumina Infinium DNA Methylation BeadArrays. Nucleic Acids Res. 41, e90-e90 (2013).

24. Ludwig, O. Blom, Gunnar: statistical estimates and transformed beta-variables. Wiley/New York, Almquist und Wiksell/Stockholm 1958; 176 S., Kr. 20,-. Biom. Z. 3, 285-285 (1961).

25. Horvath, S. et al. An epigenetic clock analysis of race/ethnicity, sex, and coronary heart disease. Genome Biol. 17, 171 (2016).

26. Li, X. et al. Longitudinal trajectories, correlations and mortality associations of nine biological ages across 20-years follow-up. elife $\mathbf{9}$, e51507 (2020).

27. Zhao, W. et al. Education and lifestyle factors are associated with DNA methylation clocks in older African Americans. Int. J. Environ. Res. Public Health 16, 3141 (2019)

28. Nevalainen, T. et al. Obesity accelerates epigenetic aging in middle-aged but not in elderly individuals. Clin. Epigenet. 9, 20 (2017).

29. Rothhammer, V. \& Quintana, F. J. The aryl hydrocarbon receptor: an environmental sensor integrating immune responses in health and disease. Nat. Rev. Immunol. 19, 184-197 (2019).
30. Juricek, L. \& Coumoul, X. The aryl hydrocarbon receptor and the nervous system. Int. J. Mol. Sci. 19, 2504 (2018).

31. the Traumatic Stress Brain Study Group. et al. An epigenome-wide association study of posttraumatic stress disorder in US veterans implicates several new DNA methylation loci. Clin. Epigenet. 12, 46 (2020).

32. INTRUST Clinical Consortium et al. Epigenome-wide meta-analysis of PTSD across 10 military and civilian cohorts identifies methylation changes in AHRR. Nat. Commun. 11, 5965 (2020).

33. Philibert, R. A., Beach, S. R. H. \& Brody, G. H. Demethylation of the aryl hydrocarbon receptor repressor as a biomarker for nascent smokers. Epigenetics 7, 1331-1338 (2012).

34. Philibert, R. A., Beach, S. R. H., Lei, M.-K. \& Brody, G. H. Changes in DNA methylation at the aryl hydrocarbon receptor repressor may be a new biomarker for smoking. Clin. Epigenet. 5, 19 (2013).

35. Zeilinger, $\mathrm{S}$. et al. Tobacco smoking leads to extensive genome-wide changes in DNA methylation. PLOS ONE 8, e63812 (2013).

36. Reynolds, L. M. et al. DNA methylation of the aryl hydrocarbon receptor repressor associations with cigarette smoking and subclinical atherosclerosis. Circ. Cardiovasc. Genet. 8, 707-716 (2015).

37. Cole, J. W. \& Xu, H. Aryl hydrocarbon receptor repressor methylation: a link between smoking and atherosclerosis. Circ. Cardiovasc. Genet. 8, 640-642 (2015).

38. Bravo-Ferrer, I. et al. Lack of the aryl hydrocarbon receptor accelerates aging in mice. FASEB J. 33, 12644-12654 (2019).

39. Quintana, F. J. et al. An endogenous aryl hydrocarbon receptor ligand acts on dendritic cells and T cells to suppress experimental autoimmune encephalomyelitis. Proc. Natl Acad. Sci. USA 107, 20768-20773 (2010).

40. Fahy, G. M. et al. Reversal of epigenetic aging and immunosenescent trends in humans. Aging Cell 18, http://pubmed.ncbi.nlm.nih.gov/ 31496122/ (2019): e13028.

41. Okazaki, S. et al. Decelerated epigenetic aging associated with mood stabilizers in the blood of patients with bipolar disorder. Transl. Psychiatry 10, 129 (2020). 